\title{
Cervical Tuberculosis: A Diagnostic Dilemma
}

\author{
${ }^{1}$ Shravya Manohar, ${ }^{2}$ Preet Agarwal, ${ }^{3} \mathrm{KS}$ Rajeswari, ${ }^{4}$ Radhika Potluri
}

\section{ABSTRACT}

This is a rare case of cervical tuberculosis, which presented as carcinoma of the cervix and gave us a diagnostic dilemma.

Keywords: Carcinoma, Cervix, Tuberculosis.

How to cite this article: Manohar S, Agarwal P, Rajeswari KS, Potluri R. Cervical Tuberculosis: A Diagnostic Dilemma. J South Asian Feder Obst Gynae 2017;9(1):66-67.

\section{Source of support: Nil}

Conflict of interest: None

Date of received: 09 September 2016

Date of acceptance: 05 November 2016

Date of publication: January 2017

\section{INTRODUCTION}

Tuberculosis of the cervix is a rare entity. It accounts for 0.1 to $0.65 \%$ of all cases of tuberculosis and 5 to $24 \%$ of all cases of genital tract tuberculosis. ${ }^{1} \mathrm{We}$, hereby, report a rare case of cervical tuberculosis with a diagnostic dilemma.

\section{CASE REPORT}

A 19-year-old nulliparous lady presented with complaints of amenorrhea and abdominal pain for the past year-anda-half. She also had complaints of postcoital bleeding and dyspareunia. She was initially diagnosed as a case of cervical carcinoma and referred to our institution for further management. Her previous menstrual cycles were regular. No past history of tuberculosis or family history of tuberculosis was present. On examination, she had no pallor and no lymphadenopathy. Chest examination was clear and per abdomen was soft with no palpable mass. On per speculum examination, there was an irregular, friable, cauliflower-like growth present on the posterior lip of the cervix. It had foul-smelling odor and bled on

${ }^{1}$ Postgraduate Student, ${ }^{2}$ Associate Professor, ${ }^{3}$ Professor ${ }^{4}$ Senior Resident

${ }^{1-4}$ Department of Obstetrics and Gynecology, Sri Ramachandra Medical College, Chennai, Tamil Nadu, India

Corresponding Author: Shravya Manohar, Postgraduate Student, Department of Obstetrics and Gynecology, Sri Ramachandra Medical College, Chennai, Tamil Nadu, India, Phone: +914428234222, e-mail: shravyamanohar1990@gmail.com touch (Fig. 1). On per rectal examination, the uterus was normal in size and mobile bilateral parametrium was free.

All investigations including hematological and biochemical were within normal limits. Chest X-ray done was normal. Pap smear showed inflammatory changes. Cervical punch biopsy done showed granulomatous inflammation (Fig. 2). Based on the diagnosis, the patient was started on antitubercular therapy (ATT) according to directly observed treatment schedule - category I isoniazid, rifampin, pyrazinamide and ethambutol.

\section{DISCUSSION}

Tuberculosis usually affects genital organs, such as the fallopian tube, endometrium, ovary, and less commonly affects the cervix. ${ }^{2}$ Clinical features are vaginal bleeding,

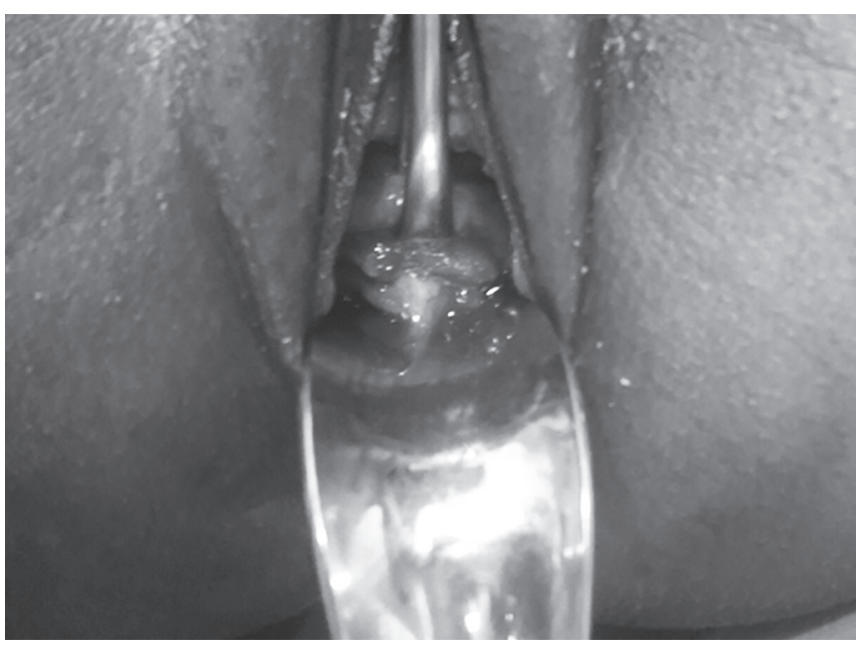

Fig. 1: Per speculum showing an irregular, friable, cauliflowerlike growth on the posterior lip of the cervix

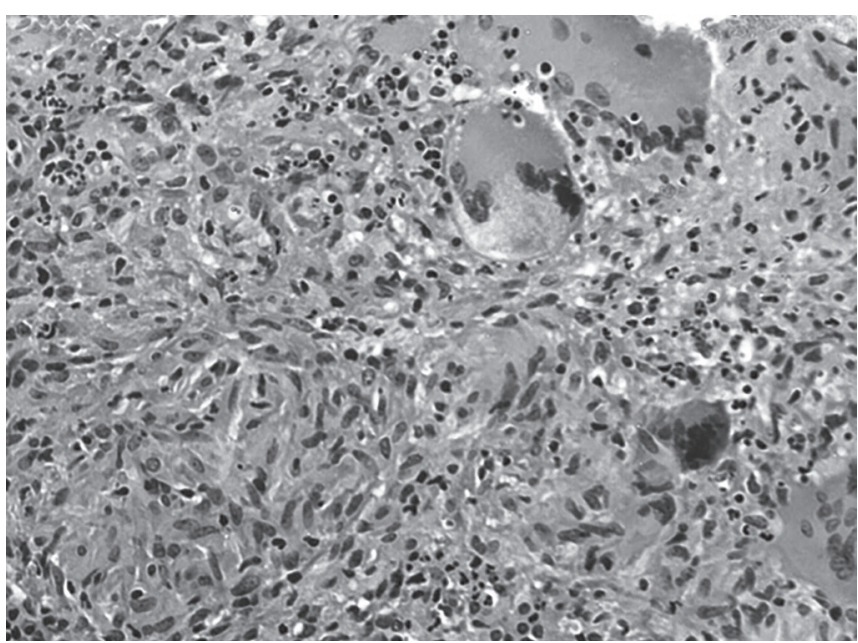

Fig. 2: Histopathology slide showing Granulomatous inflammation 
menstrual irregularities, abdominal pain, and constitutional symptoms. ${ }^{3}$ Pelvic organs may be infected from a primary focus, such as pulmonary tuberculosis by hematogenous spread. The cervix is usually affected by lymphatic or direct spread. Very rarely, cervical tuberculosis is caused by a primary infection, which may be due to causes, such as tuberculous epididymitis in the partner. ${ }^{4}$ Diagnosis is made by cervical biopsy. The gold standard for diagnosis is isolation of mycobacterium, but one-third of the cases show a negative culture. Microscopically, they present with extensive chronic inflammation with either caseating or noncaseating granulomas. Macroscopically, it shows hypertrophy of cervix or friable papillary or vegetative growth. ${ }^{5}$ The patients responded well to 6 months of standard ATT and required regular follow-up to observe the healing of the lesion. ${ }^{5}$ Fertility is generally poor after treatment, as there is subsequent endometrial and tubal involvement and healing occurs by fibrosis. The differential diagnoses are schistosomiasis, brucellosis, tularemia, and sarcoidosis. ${ }^{6}$

\section{CONCLUSION}

Diagnosis of tuberculosis cannot be made clinically and is likely to mimic carcinoma of the cervix. The confirmatory test is a cervical biopsy. In view of high incidence of tuberculosis in developing countries, there should be a high index of suspicion of tuberculosis.

\section{CLINICAL SIGNIFICANCE}

When a cervical growth is present, the differential diagnosis of tuberculous growth should also be considered.

\section{REFERENCES}

1. Lamba H, Byrne M, Goldin R, Jenkins C. Tuberculosis of the cervix: case presentation and a review of the literature. Sex Transm Infect 2002 Feb;78(1):62-63.

2. Samantaray S, Parida G, Rout N, Giri SK, Kar R. Cytologic detection of tuberculous cervicitis: a report of 7 cases. Acta Cytol 2009 Sep-Oct;53(5):594-596.

3. Singh S, Gupta V, Modi S, Rana P, Duhan A, Sen R. Tuberculosis of uterine cervix: a report of two cases with variable clinical presentation. Trop Doct 2010 Apr;40(2):125-126.

4. Jaiprakash P, Pai K, Rao L. Diagnosis of tuberculous cervicitis by Papanicolaou-stained smear. Ann Saudi Med 2013 Jan-Feb;33(1):76-78.

5. Paprikar M, Biswas M, Bhattachary S, Sodhi B, Mukhopadhyay I. Tuberculosis of cervix: case report. Med J Armed Forces India 2008 Jul;64(3):297-298.

6. Joshi PS, Shankar Vijay S, Sinha P. An unusual case of cervical tuberculosis. Online J Health Allied Sci 2011;10(1):21. 